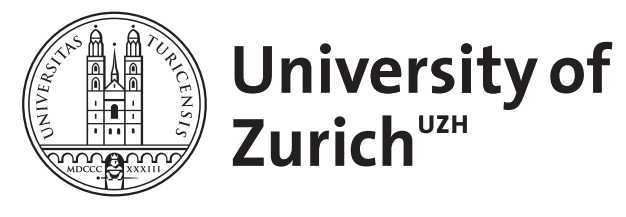

\title{
Ulcerative fungal keratitis in a Brown Swiss cow
}

\author{
Voelter-Ratson, K ; Monod, M ; Braun, Ueli ; Spiess, Bernhard M
}

\begin{abstract}
An 11-year-old Swiss Brown cow was referred to the Food Animal Department of the Veterinary Teaching Hospital in Zurich, Switzerland due to lateral recumbency of unknown origin. The animal had developed enophthalmos due to dehydration at the time of presentation. Two days after hospitalization, the cow showed blepharospasm and epiphora of the right eye and the Ophthalmology Service was consulted. Ophthalmic examination of the right eye revealed a fluorescein positive, paraxial, corneal superficial ulcer with focal edema and mild superficial neovascularization. Corneal, white, stromal infiltrates were seen at the edges of the ulcer bed. After initial treatment with topical antibiotics, an increase of corneal infiltrates was noted on re-examination 2 days later. Several punctate, fluorescein negative, stromal, white opacities were seen dorsal of the ulcer. Cytology showed fungal hyphae. Topical treatment with $2 \%$ miconazole ointment, antibiotics and K-EDTA resolved the clinical symptoms within 6 days. Fungal culture and polymerase chain reaction (PCR) identified the fungal organism as Eurotium amstelodami.
\end{abstract}

DOI: https://doi.org/10.1111/vop.12037

Posted at the Zurich Open Repository and Archive, University of Zurich ZORA URL: https://doi.org/10.5167/uzh-75775

Journal Article

Accepted Version

Originally published at:

Voelter-Ratson, K; Monod, M; Braun, Ueli; Spiess, Bernhard M (2013). Ulcerative fungal keratitis in a Brown Swiss cow. Veterinary Ophthalmology, 16(6):464-466.

DOI: https://doi.org/10.1111/vop.12037 
1 Ulcerative fungal keratitis in a cow

2 K Voelter-Ratson, 1 ....., 2 M Monod, 3, BM Spiess, 1

3 Equine Department, Vetsuisse Faculty, University of Zurich, Switzerland; 1 Bovine

4 Department, Vetsuisse Faculty, University of Zurich, Switzerland; 2 Dermatology

5 Department, University of Lausanne; 3

6

7 Address communications to:

$8 \quad$ Katrin Voelter-Ratson 
27 ABSTRACT

28
An 11-year-old Swiss Brown cow was referred to the Food Animal Department of the Veterinary Teaching Hospital in Zurich, Switzerland due to lateral recumbency of unknown origin. The animal had developed enophthalmos due to dehydration at the time of presentation. Two days after hospitalization, the cow showed blepharospasm and epiphora of the right eye and the Ophthalmology Service was consulted. Ophthalmic examination of the right eye revealed a fluorescein positive, paraxial, corneal superficial ulcer with focal edema and mild superficial neovascularization. Corneal, white, stromal infiltrates were seen at the edges of the ulcer bed. After initial treatment with topical antibiotics, an increase of corneal infiltrates was noted on re-examination 2 days later. Several punctate, fluorescein negative, stromal, white opacities were seen dorsal of the ulcer. Cytology showed fungal hyphae. Topical treatment with $2 \%$ miconazole ointment, antibiotics and K-EDTA resolved the clinical symptoms within 6 days. Fungal culture and polymerase chain reaction (PCR) identified the fungal organism as

Key Words: eye, bovine, keratomycosis, fungal, Eurotium amstelodami

\section{CASE REPORT}

An 11-year-old Brown Swiss cow was referred to the Food Animal Department of the Veterinary Teaching Hospital in Zurich, Switzerland. The cow was hospitalized because of lateral recumbency of unknown origin. A complete ophthalmic examination was performed with slit lamp biomicroscopy (Kowa SL-15; Kowa Company Ltd, Tokyo, Japan) and indirect 
ophthalmoscopy (Omega 500; Heine, Ettenheim, Germany) on the second day after hospitalization due to mild blepharospasm and epiphora. The examination of the right eye revealed a $2 \mathrm{~mm}$ fluorescein-positive, paraxial, superficial corneal ulcer with focal edema and mild superficial neovascularization. Some corneal white infiltrates in the edges of the ulcer bed were noted. Initial treatment consisted of topical triple antibiotic ointment (Neotracin, Omnivision, Switzerland) three times a day (TID). At the re-check two days later, the cow showed less blepharospasm but the amount of corneal infiltrates had increased despite antibiotic treatment. Several white, fluorescein-negative, punctate, stromal opacities were noted in an area dorsal to the ulcer. The ulcer still showed stain uptake with fluorescein dye in an area of $2 \times 2 \mathrm{~mm}$. Intraocular pressure (IOP) measured by rebound tonometry (Tonovet ; Tiolat, Espoo, Finland) was $16 \mathrm{mmHg}$ in the right eye and $18 \mathrm{mmHg}$ in the left eye. The rest of the ophthalmological examination was unremarkable. The left eye did not show any abnormalities at any time point during hospitalization. Corneal scraping of the area of white infiltrates was performed with a cytobrush. Cytology with a modified Wright-Giemsa staining (Diff-Quik, Medion Diagnostics, Switzerland) showed a cluster of epithelial cells with fungal hyphae and few neutrophilic granulocytes. The cytobrush was sent to the laboratory of the Dermatology Department of the University of Lausanne, Switzerland for fungal culture. was cultured on Sabouraud Agar and identified by PCR. E-tests (voriconazole, fluconazole, itraconazole, amphotericin B) or broth dilution (miconazole) susceptibility tests were performed for 5 antifungal drugs. MICs were defined as shown in Table 1. Treatment was initiated immediately after cytological identification of fungal organisms. Miconazole $2 \%$ ointment and K-EDTA $0.36 \%$ eye drops were administered 6 times daily and four times daily from the second day on. Antibiotic treatment was continued TID. Within 6 days after initiation of the antifungal treatment, the epithelial lesion had healed and the infiltrates disappeared. The cow was discharged 7 days after 
diagnosis of fungal keratitis with miconazole ointment TID for another 2 weeks. The cow was hospitalized again 3 weeks after discharge due to bad general condition and was euthanized 1 week later. The eye did not show any clinical symptoms at this time point.

\section{DISCUSSION}

Ulcerative keratomycosis is considered to be relatively common in humans and horses $[1,2]$.

While Infectious Bovine Keratoconjunctivitis caused by

is a common infectious disease in cattle, not much is known about keratomycosis in bovine patients [3]. A recent publication reported a case of and stromal abscess [4].To the authors' knowledge no case report exists describing an ulcerative keratitis caused by (synonym: belonging to the group of mold) in any animal species This fungal species is discussed as being a causing agent of the farmer's lung disease in humans and it might play a role in equine recurrent airway obstruction $[5,6]$. A recent publication analyzed hay samples from different European countries, which commonly contained organisms $[7,8]$. is one of the fungal species most often causing fungal keratitis in horses [9]. Fungi are ubiquitously found in hay, straw and similar materials in the environment of cows and horses. Cows showed lower numbers of fungi in the conjunctival flora of healthy animals than horses in one study [10]. A primary corneal epithelial disruption is necessary to allow the fungal organism to adhere to the corneal stroma[1]. In this case the cow was bedded on hay and straw while in lateral recumbency so we theorize that a corneal erosion caused by decreased blinking frequency or trauma due to the bad general condition and dehydration facilitated fungal adhesion. In horses, a deficient immunoreaction of the tear film or cornea to fungal organisms is theorized, the cow in this case might have had a general immunodeficiency due to its bad condition [11].

Cytology and fungal culture both revealed fungal organisms. Newer diagnostic possibilities 
101

102

103

104

like PCR allow exact identification of fungal species. Susceptibility tests were performed to establish minimal inhibitory concentrations (MIC). The importance of MIC in mycology is a frequently discussed topic as the testing cannot be easily extrapolated to the situation [12]. Yet, at present, it is the only method to determine susceptibility of fungi [2]. No MICs have been published for . Considering the MICs of the identified had good susceptibility for voriconazole, miconazole, amphotericin $\mathrm{B}_{2}$ and itraconazole while fluconazole did not seem to efficiently inhibit growth. In our case treatment with miconazole was sufficient to resolve clinical symptoms. Miconazole was chosen as it is the only antifungal drug available as a dermal ointment in Switzerland and the cost of the drug is comparatively low which makes it an excellent choice for treatment in food and fiber producing animals. Fungal keratitis should be considered as a differential diagnosis for infectious corneal ulcers in the cow.

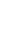

TABLE 1: MIC values of susceptibility testing.

\begin{tabular}{|l|l|}
\hline Antifungal agent & Measured \\
\hline Voriconazole & $0.032 \mathrm{~g} / \mathrm{ml}$ \\
\hline Fluconazole & $>256 \mathrm{~g} / \mathrm{ml}$ \\
\hline Itraconazole & $0.002 \mathrm{~g} / \mathrm{ml}$ \\
\hline Amphotericin B & $0.047 \mathrm{~g} / \mathrm{ml}$ \\
\hline Miconazole & $1 \mathrm{~g} / \mathrm{ml}$ \\
\hline
\end{tabular}




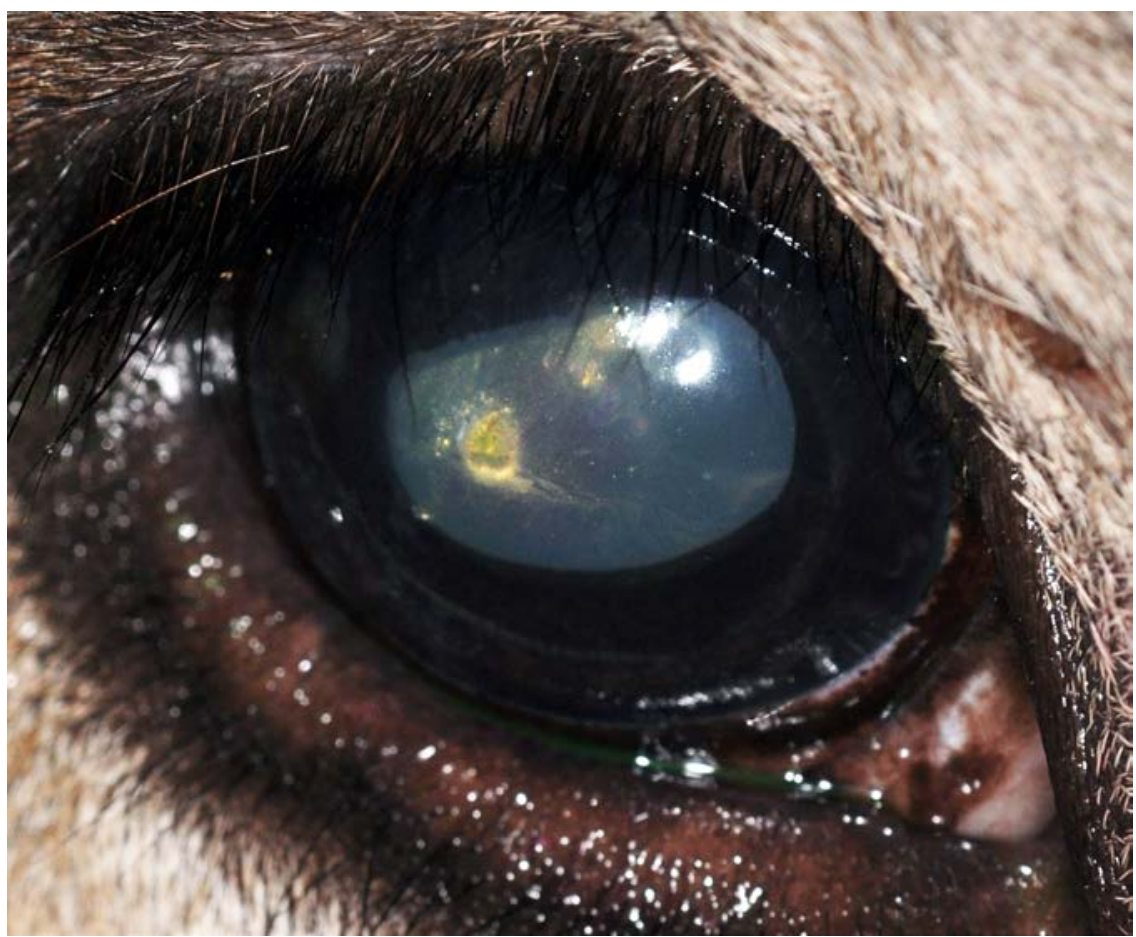

133

134 


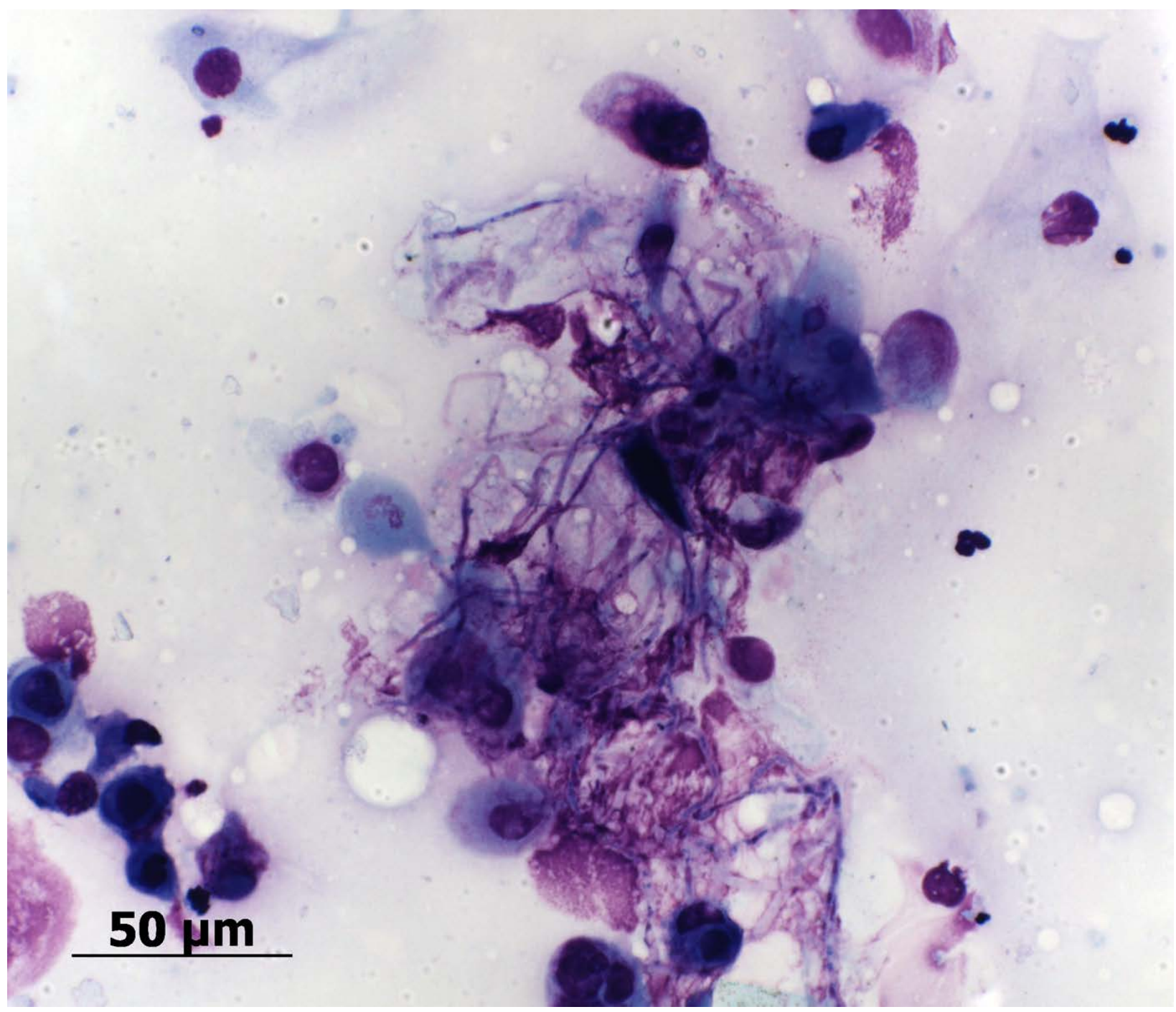

2. Lalitha, P., et al.,

(6): p. 789-93.

$$
\text { (4): p. 259-66. }
$$

1434 Elligott, C.R., et al.,

144 Ophthalmol, 2006. (3): p. 175-8.

145 5. Roussel, S., et al.,
6. Beeler-Marfisi, J., et al.,

(6): p. 682-9.

7. Radon, K., et al., Environ Med, 2002. (1): p. 41-8.

8. Roussel, S., et al.,
J Vet Intern Med, 1998.

Vet

Clin Vaccine Immunol, $2010 . \quad$ (1): p. 160-7.

Am J Vet Res, 2010.

Ann Agric 

J, 2012.

10. Samuelson, D.A., T.L. Andresen, and R.M. Gwin, J Am Vet Med Assoc, $1984 . \quad$ (10): p. 1240-2.

11. Andrew, S.E., et al.,

Equine Vet J, $1998 . \quad$ (2): p. 109-16.

12. Shapiro, B.L., et al., 